\title{
Participação dos pacientes na segurança dos cuidados de saúde: revisão sistemática
}

\author{
Participation of patients in healthcare security: a systematic review
}

Filipe Morais Figueiredo (https://orcid.org/0000-0001-7782-8808) ${ }^{1}$

Ana Maria Porcel Gálvez (https://orcid.org/0000-0001-8461-6379) ${ }^{1}$

Eugenia Gil Garcia (http://orcid.org/0000-0003-3862-6845) ${ }^{1}$

Margarida Eiras (https://orcid.org/0000-0001-5759-7336) ${ }^{2}$

${ }^{1}$ Facultad de Enfermeria Fisioterapia y Podologia, Universidad de Sevilla. C/ S. Fernando 4.41004 Sevilla España.

morais_ff@hotmail.com

${ }^{2}$ Centro de Investigação em

Saúde Pública, Universidade

Nova de Lisboa. Lisboa

Portugal.

\begin{abstract}
The scope of this article was to identify the strategies used for the participation of the patient in healthcare security in hospital and outpatient environments. It involved a systematic review of the literature based on the recommendations of the PRISMA model on the Scopus, WOS and Medline databases. The search was restricted to studies written in Portuguese, English or Spanish conducted between January 2001 and July 2016. Observational, descriptive, qualitative and/ or epidemiological studies that described a development/appliance methodology using at least one patient security improvement strategy of inclusion were included. The methodological quality of the studies was assessed using the randomized Cochrane risk-of-bias tool. Thematic analyses were performed in order to analyze the results. After the application of criteria of title, abstract analysis and exclusion, 19 studies were selected. In these studies, patient security strategies that promoted patients' active participation on patient security and information request strategies were identified. In the literature, sundry strategies promoting patient participation on healthcare security, with concrete implementation methods, as well as distinct purposes for their use, were encountered.

Key words Patient security, Patient participation, Security management
\end{abstract}

Resumo $O$ artigo objetiva identificar as estratégias utilizadas, para participação do paciente na segurança do cuidado de saúde. Revisão sistemática, norteada pelas recomendações do modelo PRISMA, nos bancos de dados: Scopus, WOS e Medline. Limitou-se a busca a estudos realizados entre janeiro de 2001 e julho de 2016, redigidos em português, inglês ou espanhol. Foram incluídos estudos observacionais, descritivos, qualitativos e/ ou epidemiológicos, que descrevessem a metodologia de elaboração elou aplicação de, pelo menos, uma estratégia de inclusão dos pacientes na melhoria da segurança dos cuidados. A qualidade metodológica dos artigos foi avaliada usando a ferramenta Cochrane. Para analisar os resultados se fez uma análise temática. Após leitura de títulos, resumos e aplicação de critérios de exclusão, 19 artigos foram selecionados. Nestes se identificam estratégias de mobilização dos pacientes para a segurança dos cuidados, estratégias para promover a participação ativa dos pacientes na segurança dos cuidados e estratégias de solicitação de informação ao paciente sobre a segurança dos cuidados. Há na literatura diversas estratégias que promovem a participação do paciente na segurança dos cuidados, que têm formas e métodos concretos de implementação, bem como objetivos distintos para o seu uso.

Palavras-chave Segurança do paciente, Participação do paciente, Gerenciamento de segurança. 


\section{Introdução}

O público espera que a segurança seja uma prioridade dentro dos serviços de saúde, no entanto, é preconizado que a prestação de cuidados de saúde envolve potenciais riscos para a segurança do paciente ${ }^{1}$, existindo uma grande diversidade de estudos publicados em diversos países que relataram a magnitude e extensão dos danos causados aos pacientes ${ }^{2-6}$. Assim, a segurança do paciente tem recebido cada vez mais atenção pelos decisores políticos, profissionais de saúde, e investigadores.

A segurança do paciente pode ser definida sucintamente, como "a redução do risco de danos desnecessários relacionados com os cuidados de saúde, para um mínimo aceitável”’

A estratégia para reduzir os danos e promover a segurança do paciente tem-se centrado principalmente na promoção de transformações dentro dos sistemas de saúde, em alterações do comportamento profissional e nos sistemas de notificação de incidentes ${ }^{3,8,9}$.

No decorrer da última década, se tem vindo a incluir gradualmente os pacientes na prevenção de danos e promoção da segurança do paciente, talvez devido a um maior foco na centralização dos cuidados no cidadão, comprovando-se assim a necessidade de colaboração de todos os atores ligados aos cuidados de saúde, de modo a contribuir para a melhoria da segurança do paciente ${ }^{1,3}$.

Neste sentido, se tem evidenciado a necessidade de promover a participação do paciente na melhoria da segurança dos cuidados de saú$\mathrm{de}^{1,3,10}$, se conceituando "participação do paciente" como a integração do mesmo na tomada de decisão em tudo que possa influenciar a sua saúde, podendo contribuir ativamente numa ampla gama de atividades, desde a formulação de planos de tratamento, até à formulação de políticas de segurança ${ }^{10,11}$.

Promover a participação do paciente na melhoria da segurança dos cuidados de saúde, implica a integração deste na tomada de decisão, de forma consciente e informada, sobre ações que podem afetar a segurança dos cuidados de saúde. Podendo assim, o paciente participar na melhoria da segurança dos cuidados através de uma vasta gama de ações, que vão desde ações solicitadas pelo sistema/serviço ao paciente, até à participação ativa e mobilização dos pacientes para a promoção e melhoria da segurança dos cuidados de saúde ${ }^{12}$.

No entanto, há pouca investigação sobre a utilização da informação fornecida pelos pacien- tes, assim como falta investigação sobre a aceitação desta participação por parte dos mesmos ${ }^{3}$. Segundo literatura científica recente há determinados temas em que os pacientes não estão dispostos a participar na tomada de decisão, e que tal resolução pode ser tomada devido a diversos fatores ${ }^{10,13-15}$. Há evidência que indica que os pacientes estão dispostos e são capazes de participar em estratégias de prevenção do erro ${ }^{16}$, e que têm potencial para melhorar a sua seguranç $c^{8,17,18}$. Está assim patente, a necessidade de conhecer e compreender melhor as formas como pode o usuário participar na melhoria da segurança dos cuidados de saúde.

Existe uma panóplia de atividades e/ou intervenções que permitem a participação do paciente/usuário na segurança dos cuidados ${ }^{19}$, que podem ser de iniciativa do próprio paciente/ usuário ou do sistema/serviço, que apresentam características e metodologias próprias, bem como objetivos distintos para a sua aplicação, e que devem ser usadas de forma adequada ao contexto da instituição ou serviço de saúde onde se pretende aplicá-la ${ }^{12}$. Ainda assim, é escassa a bibliografia que agregue, explicite e exemplifique estas diferentes estratégias de promoção da participação dos pacientes na segurança dos cuidados.

Assim, os métodos e estratégias para promover a participação do paciente na segurança dos cuidados podem dividir-se em três tipos, que têm formas e métodos concretos de implementação, bem como objetivos distintos: estratégias de mobilização dos pacientes para a segurança dos cuidados; estratégias que pretendem promover a participação ativa do paciente na prevenção de incidentes; e estratégias que pretendem solicitar e utilizar a informação relevante que os pacientes podem fornecer ${ }^{9,12}$.

As estratégias de mobilização dos pacientes para a segurança dos cuidados, cujos sistemas de notificação de incidentes são exemplo, visam desenvolver nos pacientes a necessidade de partilha de problemas de segurança que possam ter sofrido ou presenciado, para evitar que esses problemas se repitam ${ }^{12}$.

As estratégias que pretendem promover a participação ativa do paciente na prevenção de incidentes de segurança, almejam motivar o paciente a participar ativamente na melhoria da segurança dos cuidados, nomeadamente indicando-lhes formas de participação e promovendo a sua capacitação e empoderamento. São exemplo deste tipo de estratégia: guias de participação ativa dos pacientes, cartazes informativos, folhetos, vídeos informativos, entre outros ${ }^{12}$. 


\section{tionários ${ }^{12}$.}

É objetivo deste trabalho, identificar as estratégias utilizadas, de participação do paciente na segurança do cuidado de saúde.

\section{Métodos}

Trata-se de um estudo de revisão sistemática de literatura, que seguiu as orientações propostas no checklist Preferred Reporting Items for Systematic Reviews and Meta-Analyses (PRISMA) ${ }^{20}$. A pergunta que direcionou a revisão foi: "Que estratégias de participação do paciente na segurança dos cuidados de saúde estão sendo utilizadas?"

\section{Estratégia de busca}

A estratégia de buscas foi criada para a base de dados Scopus, e posteriormente adaptada à WOS (Web of Science) e Medline, tendo sido desenvolvida pela equipe de investigação, utilizando diversas combinações de termos do Medical Subject Heading Terms (MeSH) que indexam os artigos, combinados com outros termos contidos em títulos e resumos (Quadro 1). Todas as buscas nas bases de dados foram conduzidas entre os meses de janeiro e julho de 2016, pelo que estudos publicados até esta data foram elegíveis para inclu- são, definindo um limite temporal entre janeiro de 2001 e julho de 2016 e restringindo as línguas de pesquisa ao inglês, espanhol e português. Não foi feita qualquer restrição em termos de país.

Para todos os estudos identificados como relevantes após o processo de seleção foi utilizada a técnica da bola de neve com pesquisas de citações, através do ISI Web of Knowledge, pela qual não houve incremento de mais estudos.

Foi criada uma base de dados utilizando o programa Mendeley, para armazenar e gerir todas as referências encontradas.

\section{Critérios de elegibilidade}

Foram incluídos todos os estudos observacionais, descritivos, qualitativos e/ou epidemiológicos, realizados em âmbito hospitalar ou nos cuidados ambulatórios que descrevessem a metodologia de elaboração e/ou aplicação de, pelo menos, uma estratégia de inclusão dos pacientes na melhoria da segurança dos cuidados, sendo que estas estratégias se categorizaram em: estratégias de mobilização dos pacientes para a segurança dos cuidados; estratégias para promover a participação ativa dos pacientes na prevenção de incidentes de segurança; e estratégias de solicitação de informação ao paciente sobre a segurança dos cuidados.

Os estudos deveriam encontra-se dentro do limite temporal previamente definido e redigidos em inglês, espanhol ou português. Sendo que apenas foram incluídos estudos a que se teve acesso à totalidade do artigo e estratégia(s) nele utilizada(s). Nos artigos que não se encontravam totalmente disponíveis, optou-se por contatar os seus autores por e-mail, sendo que todos os contatados atenderam à solicitação. Excluíram-se

Quadro 1. Estratégia de buscas desenvolvida para a base de dados Scopus e adaptada paras as restantes bases de dados.

\begin{tabular}{|c|c|}
\hline $\begin{array}{c}\text { Base de } \\
\text { datos }\end{array}$ & Descriptor \\
\hline \multirow[t]{8}{*}{ Scopus } & patient $^{\star}$ participation ${ }^{\star}$ AND patient ${ }^{\star}$ safety AND safety management \\
\hline & patient $^{\star}$ safety AND patient ${ }^{\star}$ participation ${ }^{\star}$ AND $\left(\right.$ patient $^{\star}$ report $^{\star}$ OR patient $^{\star}$ experience $\left.^{\star}\right)$ \\
\hline & patient $^{\star}$ participation $^{\star}$ AND adverse event* AND patient* ${ }^{\star}$ safety \\
\hline & Primary Care AND Patient perception AND Adverse event \\
\hline & $\left(\right.$ Hospital $^{\star}$ OR Ward $\left.{ }^{\star}\right)$ AND Patient ${ }^{\star}$ perception ${ }^{\star}$ AND Adverse event ${ }^{\star}$ \\
\hline & $\left(\right.$ Hospital $^{\star}$ OR Ward $\left.{ }^{\star}\right)$ AND Patient ${ }^{\star}$ perception$^{\star}$ AND patient ${ }^{\star}$ safety \\
\hline & patient $^{\star}$ participation $^{*}$ AND patient ${ }^{\star}$ safety AND (questionnaire OR interviews OR Focal Group) \\
\hline & $\begin{array}{l}\text { patient }^{\star} \text { participation }{ }^{\star} \text { AND patient* safety AND safety management AND (questionnaire OR } \\
\text { interviews OR Focal Group) }\end{array}$ \\
\hline
\end{tabular}


do estudo, artigos de revisão sistemática de literatura.

\section{Seleção dos estudos}

Numa primeira fase realizou-se uma leitura dos títulos e/ou abstracts aplicando os critérios de seleção. Após esta fase, dois revisores independentes executaram uma leitura completa de todos os artigos selecionados, aplicando os critérios de seleção, sendo que qualquer desentendimento era resolvido por consenso com um terceiro revisor.

A qualidade metodológica de cada estudo selecionado foi verificada pela ferramenta Cochra$\mathrm{ne}^{21}$, que utiliza sete princípios para avaliar o risco de viés de cada estudo: randonização adequada; ocultação de alocação; cegamento dos participantes, cegamento do avaliador dos resultados; integridade dos resultados, dados incompletos; relatórios seletivos dos resultados; e outras fontes de viés ${ }^{22}$. Para este estudo foi feita uma adaptação desta ferramenta, que pode ser vista no Quadro 2, na qual se utilizaram cinco critérios para avaliar o risco de viés de cada estudo.

\section{Análise dos estudos}

Para analisar os estudos selecionados, se realizou uma análise temática, para avaliar semelhanças e diferenças entre os mesmos. A análise temática é um método apropriado para resumir e organizar os resultados de um conjunto de estudos que engloba estudos quantitativos e qualitativos $^{23}$. A ênfase desta análise centrou-se na percepção das estratégias de participação dos pacientes na segurança dos cuidados de saúde presentes em cada estudo, nas semelhanças e diferenças perceptíveis na utilização da mesma estratégia e na busca de um padrão de complementariedade entre as estratégias utilizadas nos diferentes estudos.

Esta abordagem interpretativa envolveu a leitura e releitura dos estudos por dois revisores e sempre que foi necessário algum esclarecimento, foram contatados os autores dos diferentes estudos analisados.

\section{Resultados}

\section{Identificação dos instrumentos}

Os resultados das pesquisas encontram-se sumarizados no fluxograma PRISMA da Figura 1. $\mathrm{Na}$ pesquisa inicial foram identificados um total de 11593 estudos, dos quais 2898 se encontravam duplicados. Após a primeira fase de seleção, em que foram analisados os títulos de todos os estudos e os resumos de 95 estudos, apuraram-se 49 estudos. Na sequência da segunda fase de seleção, na qual foram analisados os 49 estudos na totalidade do seu conteúdo, foram selecionados 19 estudos, nos quais foram aplicados um total de 23 estratégias de participação dos pacientes na segurança na prestação de cuidados de saúde.

\section{Caracterização dos estudos identificados}

O Quadro 3 agrega uma súmula dos objetivos e resultados de cada estudo, bem como do âmbito de utilização e/ou elaboração dos diferentes

Quadro 2. Avaliação do risco de viés de cada artigo.

\begin{tabular}{|c|c|c|c|c|c|c|c|c|c|c|c|c|c|c|c|c|c|c|c|}
\hline & 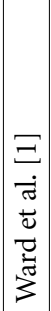 & 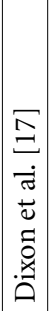 & 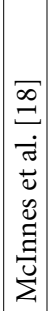 & 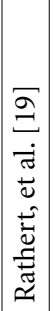 & 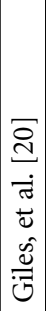 & 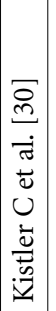 & 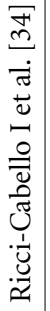 & 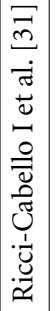 & 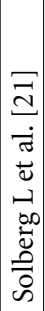 & 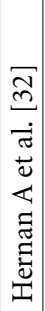 & 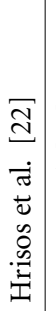 & 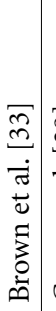 & 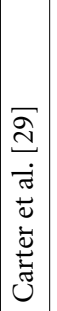 & 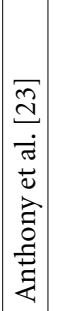 & 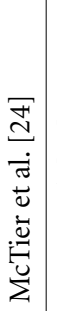 & 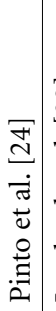 & 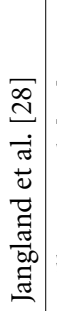 & 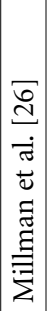 & 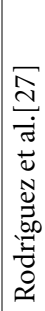 \\
\hline Gerador de sequência (viés de seleção) & + & $?$ & - & $?$ & + & - & - & $?$ & $?$ & - & - & - & - & + & - & + & - & - & - \\
\hline Desconhecimento dos participantes (viés de realização) & $?$ & $?$ & + & $?$ & + & + & + & + & + & - & $?$ & - & + & + & ? & + & + & $?$ & $?$ \\
\hline Desconhecimento do avaliadores (viés de deteção) & $?$ & + & + & + & + & + & + & + & + & $?$ & $?$ & + & + & + & $?$ & + & $?$ & $?$ & + \\
\hline Dados de resultados incompletos (viés de atrito) & + & + & + & + & + & + & + & + & + & + & + & + & + & $?$ & + & + & + & + & + \\
\hline Resultados seletivos (viés de notificação) & $?$ & + & + & + & + & + & + & $?$ & + & + & + & + & + & + & + & + & + & $?$ & + \\
\hline
\end{tabular}

Legenda: + Baixo Risco - Risco de Viés ? Não Claro. 
Figura 1. Fluxograma PRISMA, com as diferentes fases da seleção dos artigos.

instrumentos identificados nos estudos selecionados.

A Tabela 1 apresenta uma caracterização generalizada de todos os estudos selecionados. Podese constatar que os países onde foram elaborados mais estudos (de entre os selecionados) foram o Reino Unido e os Estados Unidos da América com 6 estudos cada um, sendo que os estudos provenientes de países anglo-saxônicos representam $89 \%$ dos estudos selecionados, tendo os outros dois sido elaborados na Espanha e na Suécia.

Nos estudos selecionados verificou-se que esta temática tem sofrido uma evolução significativa nos últimos anos, pois $45 \%$ desses estudos foram elaborados nos últimos três anos e $80 \%$ nos últimos seis anos, sendo o estudo mais antigo datado de 2002.

A maioria dos estudos selecionados (59\%) foram realizados em âmbito hospitalar, ou incluíram o meio hospitalar ${ }^{2,24-35}$, sendo que destes, mais de metade se aplicaram, ou se pretendem aplicar num serviço específico do meio hospita- lar. Três dos estudos selecionados incluem mais de um âmbito, sendo que dois se aplicam em hospitais e cuidados de saúde primários ${ }^{28,34}$ e outro estudo se aplica em cuidados de saúde primários e farmácias ${ }^{36}$.

Nos 19 estudos foi possível observar a utilização e/ou elaboração de 23 estratégias de inclusão do paciente na segurança dos cuidados de saúde. Identificaram-se assim: dezessete estratégias que permitem aos sistemas/instituições solicitar informação relevante que os pacientes podem fornecer, dividindo-se em oito questionários $^{2,24-26,28,37-39}$, cinco entrevistas ${ }^{27,29,31,34,40}$ e quatro grupos focais ${ }^{24,34,36,41}$; três estratégias que visam a promoção da participação ativa dos pacientes na prevenção de incidentes de segurança dos cuidados de saúde, nomeadamente dois vídeos ${ }^{30,32}$ e um folheto informativo ${ }^{35}$; e três estratégias de mobilização dos pacientes para a segurança dos cuidados de saúde quer através da disponibilização de um sistema de notificação de incidentes, para os pacientes, que se verificou em dois estu- 


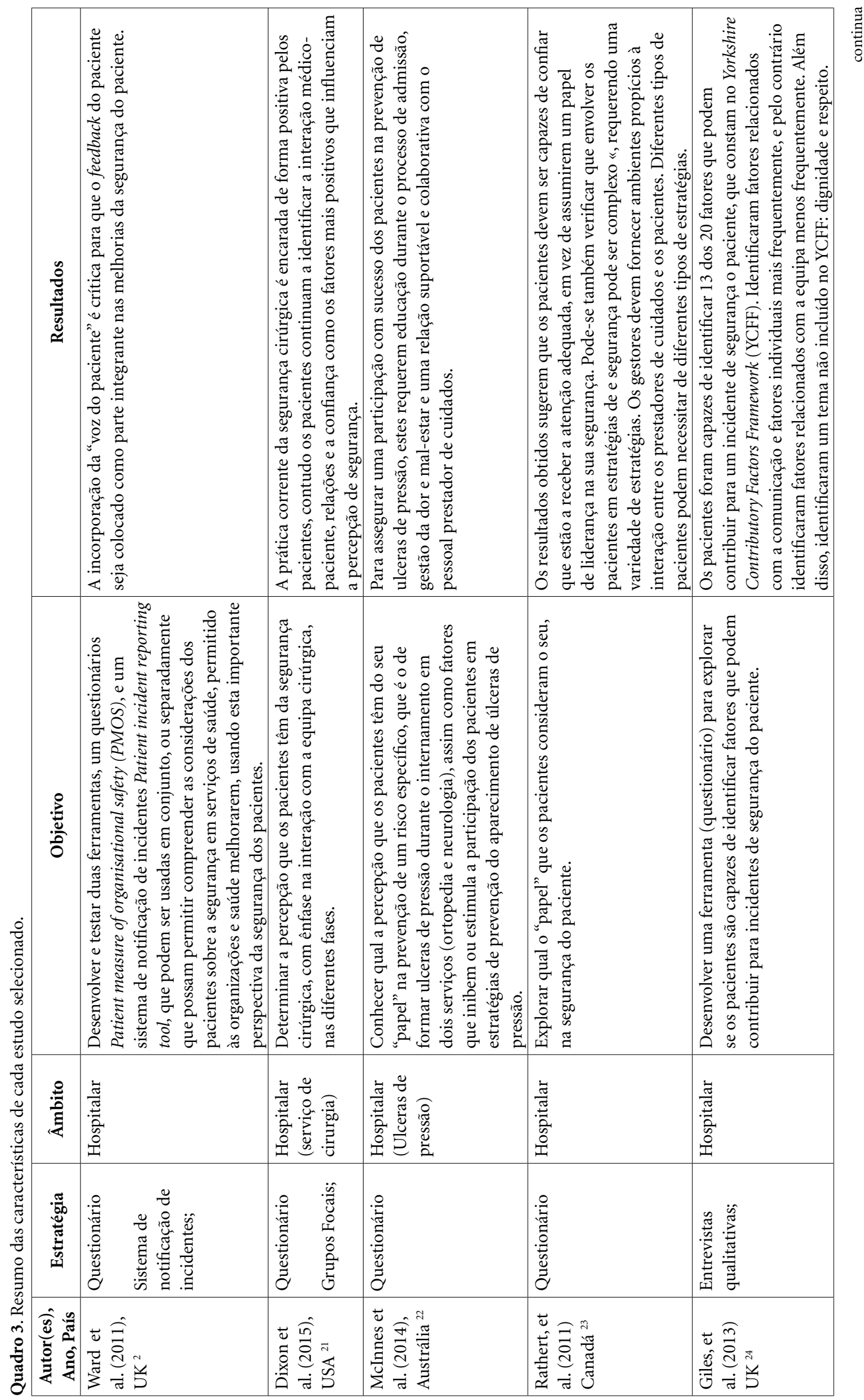




\begin{tabular}{|c|c|c|c|c|c|c|}
\hline 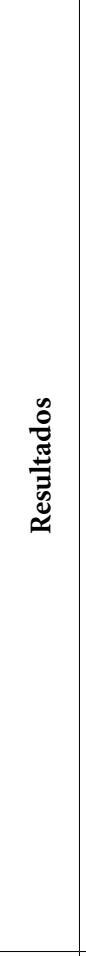 & 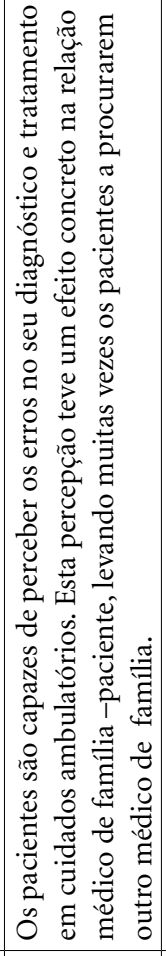 & 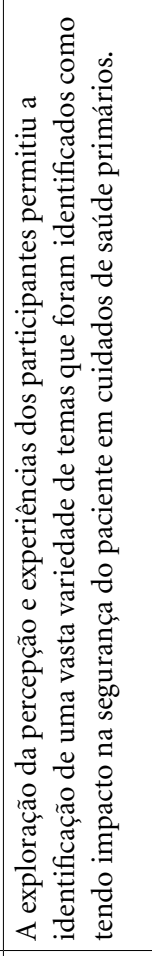 & 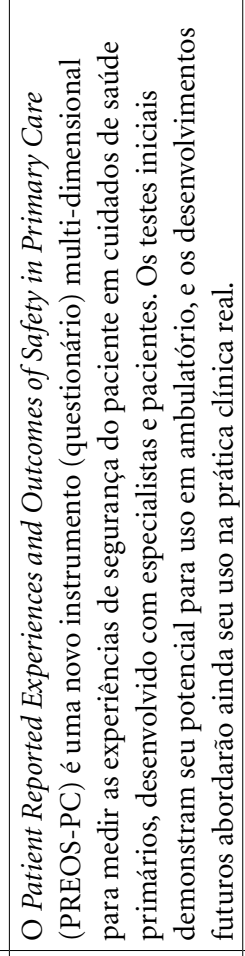 & 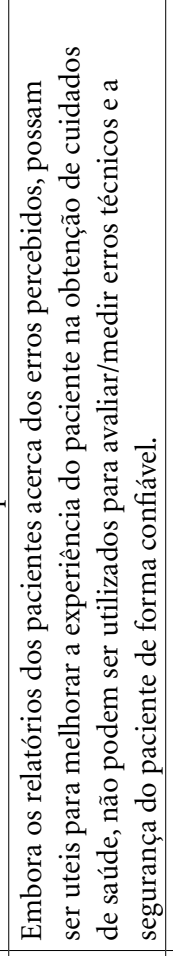 & 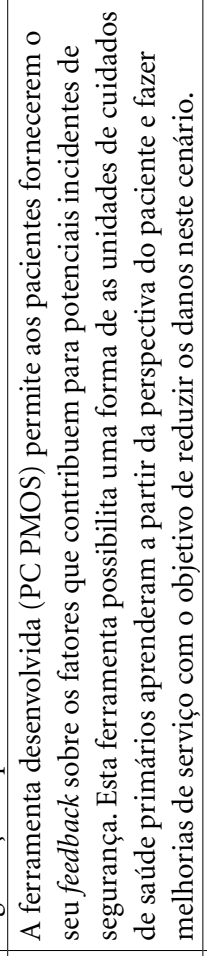 & 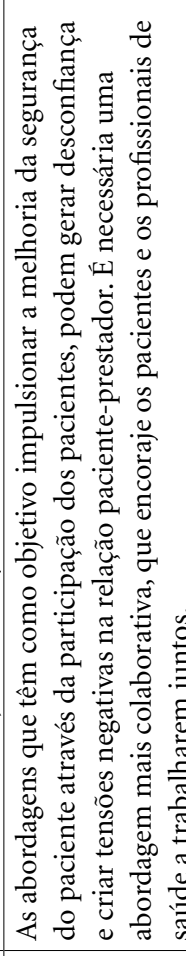 \\
\hline & 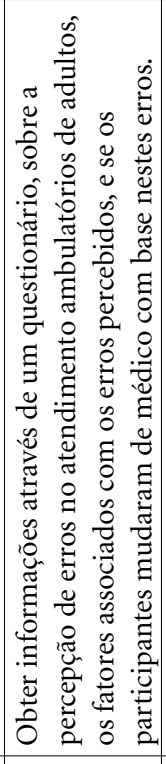 & 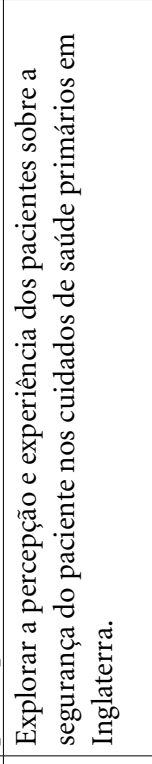 & 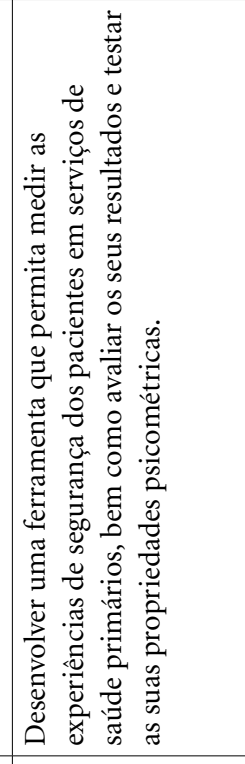 & 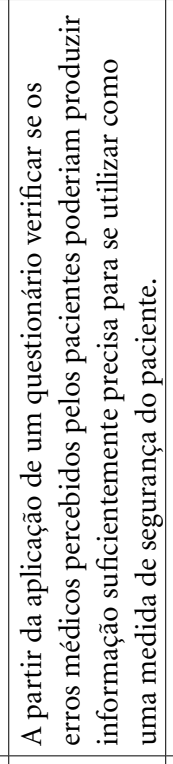 & 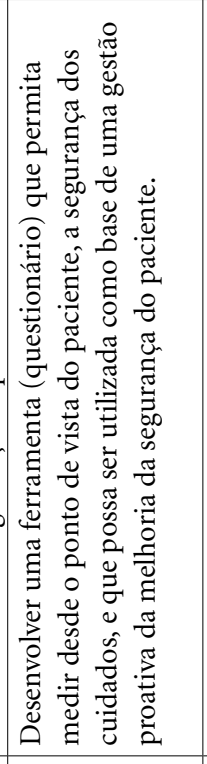 & 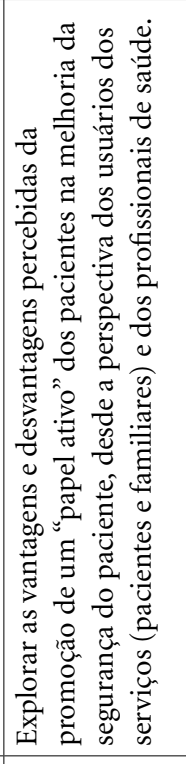 \\
\hline 韋 & 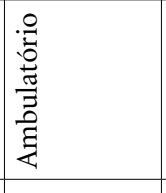 & 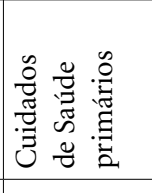 & 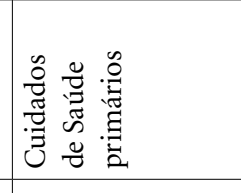 & 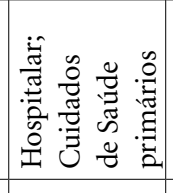 & 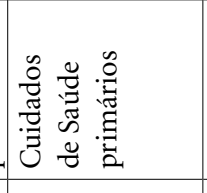 & 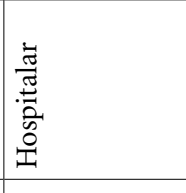 \\
\hline 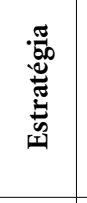 & 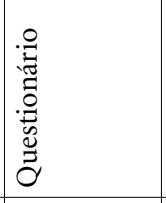 & 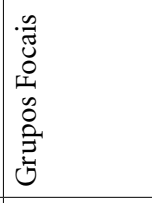 & 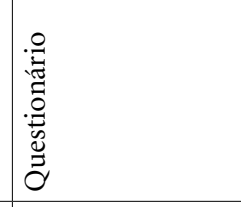 & 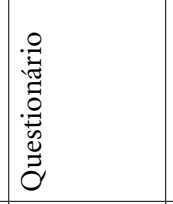 & 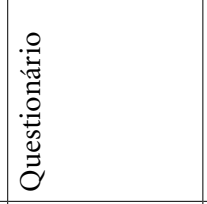 & 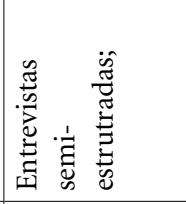 \\
\hline 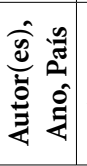 & 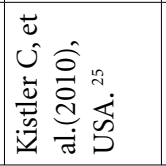 & 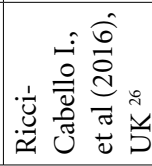 & 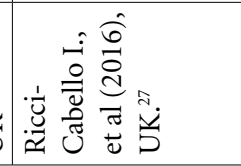 & 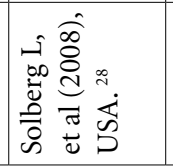 & 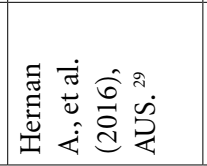 & 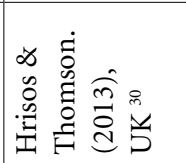 \\
\hline
\end{tabular}




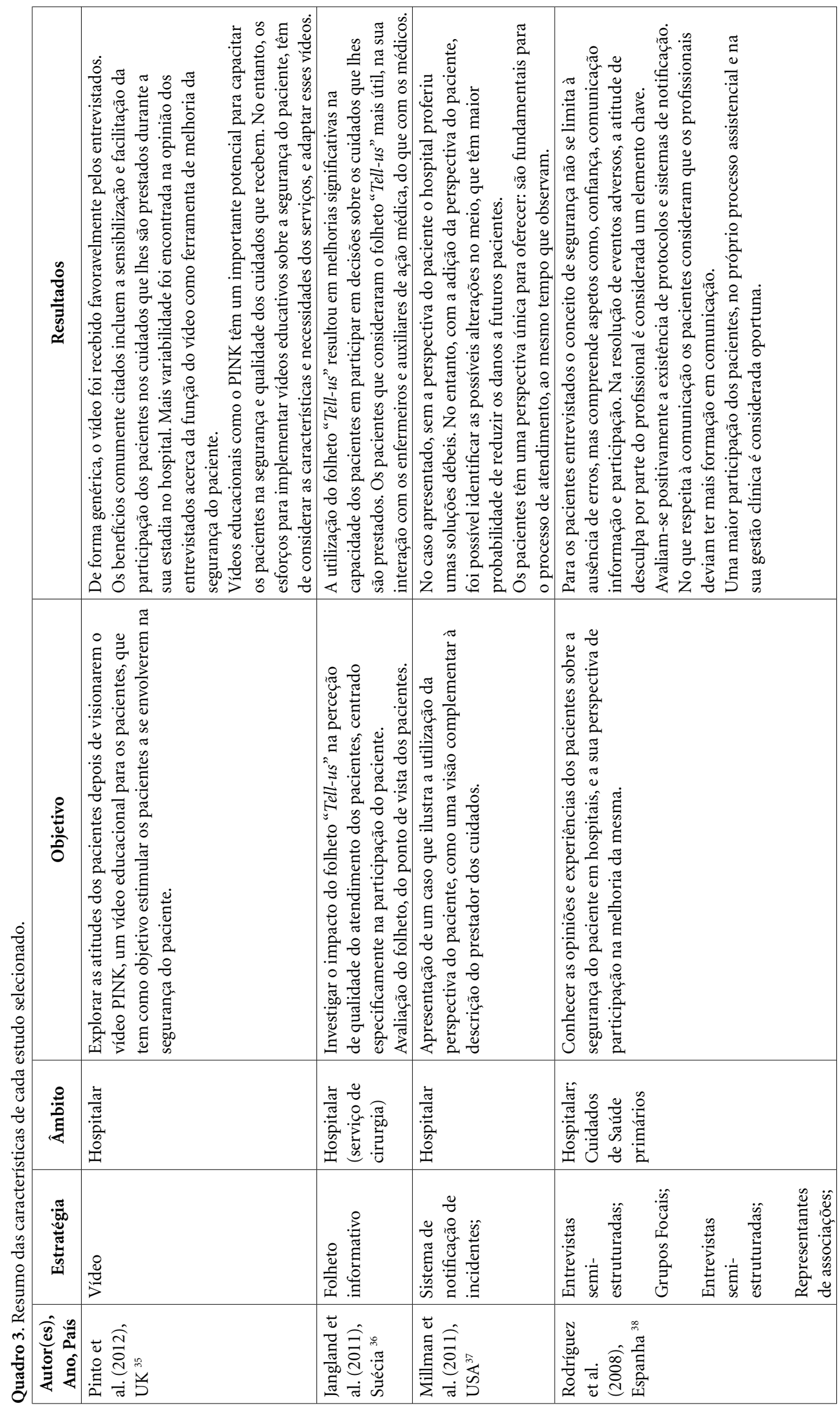


Tabela 1. Caracterização dos estudos selecionados.

\begin{tabular}{|c|c|c|}
\hline & & $\begin{array}{c}\text { No de } \\
\text { instrumentos } \\
(\%)\end{array}$ \\
\hline \multicolumn{3}{|c|}{$\begin{array}{l}\text { País no qual o instrumento foi } \\
\text { desenvolvido/aplicado }\end{array}$} \\
\hline \multicolumn{2}{|l|}{ UK } & $6(32)$ \\
\hline \multicolumn{2}{|l|}{ USA } & $6(32)$ \\
\hline \multicolumn{2}{|l|}{ Austrália } & $4(21)$ \\
\hline \multicolumn{2}{|l|}{ Canadá } & $1(5)$ \\
\hline \multicolumn{2}{|l|}{ Suécia } & $1(5)$ \\
\hline \multicolumn{2}{|l|}{ Espanha } & $1(5)$ \\
\hline \multicolumn{3}{|c|}{ Ano de publicação } \\
\hline \multicolumn{2}{|c|}{$2001-2004$} & $1(5)$ \\
\hline \multicolumn{2}{|l|}{$2005-2008$} & $3(16)$ \\
\hline \multicolumn{2}{|l|}{$2009-2012$} & $7(37)$ \\
\hline \multicolumn{2}{|c|}{2013 - Atualidade } & $8(42)$ \\
\hline \multicolumn{3}{|c|}{ Âmbito de aplicação } \\
\hline \multicolumn{2}{|c|}{ Hospitalar (geral) } & $7(32)$ \\
\hline \multicolumn{2}{|c|}{ Hospitalar (serviços específicos) } & $6(27)$ \\
\hline \multicolumn{2}{|c|}{ Cuidados de Saúde Primários } & $8(36)$ \\
\hline \multicolumn{2}{|c|}{ Farmácia } & $1(5)$ \\
\hline \multicolumn{3}{|c|}{ Tipos de instrumentos utilizados } \\
\hline \multicolumn{2}{|c|}{ Questionário } & $8(35)$ \\
\hline Entrevistas & Estruturadas & $2(9) \quad 5(22)$ \\
\hline & $\begin{array}{l}\text { Semi- } \\
\text { estruturadas }\end{array}$ & $3(1)$ \\
\hline Grupos Foca & & $4(17)$ \\
\hline Vídeos & & $2(9)$ \\
\hline Folhetos & & $1(4)$ \\
\hline $\begin{array}{l}\text { Sistema de } \mathrm{n} \\
\text { incidentes }\end{array}$ & ícação de & $2(9)$ \\
\hline $\begin{array}{l}\text { Mobilização } \\
\text { utentes }\end{array}$ & associações de & $1(4)$ \\
\hline
\end{tabular}

$\operatorname{dos}^{2,33}$, quer através de inclusão de associações de usuários na identificação de situações passiveis de melhoria, para aumentar a segurança do paciente, que se verificou num estudo ${ }^{34}$.

\section{Estratégias que permitem aos sistemas/instituições solicitar informação relevante que os pacientes podem fornecer}

Este tipo de estratégia pode ser dividida de acordo com os dados fornecidos pelos instrumentos utilizados, havendo assim estratégias que fornecem dados quantitativos, como é o caso dos questionários, e estratégias que fornecem dados qualitativos, como as entrevistas e os grupos focais ${ }^{12}$.

\section{Questionários}

Nos oito questionários identificados, quatro (50\%) têm como principal foco, facultar a perspectiva do paciente sobre a segurança na prestação de cuidados ${ }^{2,24,39,41}$, dois têm como objetivo primordial perceber o "papel" que os pacientes consideram poder ter na segurança do paciente $^{25,28}$, enquanto os outros dois visam identificar os erros dos profissionais, que os pacientes conseguem detectar, e que podem afetar a segurança do paciente ${ }^{26,37}$.

Sete destes questionários estão a ser aplicados nos respectivos estudos, enquanto um está em fase de desenvolvimento e validação ${ }^{2}$.

No que concerne ao âmbito de utilização cinco dos questionários são utilizados em âmbito hospitalar, e quatro em cuidados de saúde primários, sendo que dois questionários são utilizados nos dois âmbitos. Em relação aos questionários a ser utilizados em meio hospitalar, há dois que se são para ser utilizados a um nível geral, e três para serem aplicados em serviços específicos.

Quatro dos questionários identificados apresentam fortes propriedades psicométricas ${ }^{2,3,39,41}$.

De entre os questionários identificados, dois (25\%) foram elaborados para serem utilizados de modo complementar a outra estratégia de participação do paciente na segurança da prestação de cuidados de saúde $e^{2,24}$.

O tipo de questionários que se utiliza depende do objetivo do estudo e do tipo de avaliação que se pretenda fazer, pelo que se verificou que uns estudos utilizavam questionários de opinião, onde se pretendeu obter a valorização do paciente segundo a sua percepção de segurança, e questionários tipo relatórios, onde se pretende obter informação do paciente sobre se ocorreram determinados aspetos concretos.

\section{Grupos focais}

Foram identificados 4 estudos em que se utilizaram grupos focais como estratégia de envolvimento dos pacientes na segurança na prestação de cuidados, sendo que em 3 se utilizavam os grupos focais com o intuito de obter a percepção e experiência dos pacientes sobre a segurança dos cuidados $^{24,36,38}$, no outro estudo a utilização de grupos focais como finalidade, identificar fatores de motivação para os pacientes participarem ativamente na segurança dos cuidados ${ }^{34}$.

Os grupos focais, são provavelmente a melhor maneira de averiguar os assuntos a incluir num questionário de qualidade percebida ${ }^{12}$, daí que um dos estudos utilize os grupos focais para a percepção de temas, que os pacientes entendam afetar a segurança do paciente, para os incluir 


\section{Entrevistas}

Nos estudos identificados, cinco deles utilizam entrevistas como estratégias de participação do paciente na segurança da prestação de cuidados, sendo que três utilizam entrevistas semi-estruturadas $\mathrm{s}^{29,34,40} \mathrm{e}$ dois utilizam entrevistas estruturadas $^{27,31}$.

As entrevistas são um método de pesquisa qualitativo muito utilizado para obter conhecimento detalhado sobre determinadas temáticas, pelo que também podem ser utilizadas para a percepção de temas que pacientes entendam que afetam a segurança do paciente para os incluir posteriormente num questionário ${ }^{12}$.

As entrevistas foram utilizadas nos diversos estudos identificados com distintos objetivos, tais como: Identificação de fatores que podem provocar um incidente de segurança; Identificação de formas de incorporação dos pacientes na segurança dos cuidados de saúde; Averiguação de vantagens da incorporação dos pacientes na segurança dos cuidados de saúde; e Definição do conceito de segurança para o paciente.

\section{Estratégias de promoção da participação ativa dos pacientes na prevenção de incidentes de segurança na prestação de cuidados}

Foram identificados três estudos em que se utilizaram este tipo de estratégia. Em dois estudos foram utilizados vídeos ${ }^{30,32}$, em outro estudo utilizou-se um Guia para a participação ativa do paciente ${ }^{35}$. Nos três casos estas estratégias foram utilizadas com o intuito de educar os pacientes para a segurança dos cuidados de saúde e os motivar a participarem ativamente. Em todos estes estudos o efeito da aplicação deste tipo de estratégia foi avaliado através de questionários elaborados para esse intuito.

\section{Estratégias de mobilização dos pacientes para a segurança dos cuidados de saúde}

Dos três estudos identificados que utilizam este tipo de estratégia, dois utilizam ou relatam

a elaboração de um sistema de notificação de incidentes de segurança para os pacientes ${ }^{2,33}$, enquanto outro mobiliza líderes de organizações de utentes para participarem em entrevistas semi-estruturadas ${ }^{34}$.

Os estudos que relatam a elaboração e utilização de um sistema de notificação de incidentes para os pacientes têm como objetivo que os sistemas/instituições retirem ilações e ensinamentos das experiências relatadas pelos pacientes. $\mathrm{O}$ estudo que utiliza as entrevistas semi-estruturadas a líderes de organizações de utentes, pretende através destas perceber as experiências sobre a segurança na prestação de cuidados que essas organizações têm conhecimento, e assim envolvê-las também na melhoria contínua do sistema.

Em dois destes estudos estas estratégias são complementares a outras estratégias de participação dos pacientes na segurança dos cuidados de saúde ${ }^{2,34}$.

\section{Discussão}

Nesta revisão sistemática da literatura foram identificadas 23 estratégias de inclusão e participação do paciente na segurança do paciente, divididas por 19 diferentes estudos. A maioria destes instrumentos/estratégias foram desenvolvidos em estudos cujo principal objetivo não era o desenvolvimento da estratégia em si, mas sim a obtenção de dados concretos que se pretendiam alcançar a partir destas. Conseguindo-se, no entanto, verificar a/s estratégia/s utilizada em cada estudo e classificá-la quanto á sua tipologia.

A maioria das estratégias identificadas eram estratégias que permitem aos sistemas/instituições solicitar informação relevante que os pacientes podem fornecer (por meio de questionários, entrevistas, grupos focais), talvez devido ao fato destas estratégias estarem relativamente bem desenvolvidas e serem razoavelmente compreendidas dentro dos sistemas, para além de possibilitarem dados concretos, que, no entanto, desacompanhados e descontextualizados podem ser de escassa utilidade ${ }^{12}$.

\section{Forças e limitações}

A principal força desta revisão sistemática reside no fato de realizar uma compilação de todas as estratégias de participação do paciente na segurança dos cuidados, executando um levantamento sistemático de todas as estratégias elaboradas e/ou aplicadas descritas na literatura, incluindo tanto as de iniciativa do próprio usuário 
como as de iniciativa do sistema/serviço, organizando estas estratégias em três tipos, consoante as mesmas tivessem como objetivo promover a participação ativa dos pacientes na: sua segurança, mobilizar os pacientes para a sua segurança ou solicitar e utilizar a informação relevante que os pacientes podem fornecer.

No que concerne às principais limitações da presente revisão sistemática, pode-se apontar a incapacidade de verificação das propriedades psicométricas das estratégias utilizadas nos diferentes estudos, bem como à impossibilidade de observar uma justificativa para a escolha das estratégias de participação dos pacientes na segurança do paciente, utilizadas em alguns estudos.

\section{Sumário da evidência}

A maioria das estratégias identificadas nesta revisão sistemática foram publicadas nos últimos seis anos ${ }^{2,24-27,29,31-33,35,36,38,39,41}$, o que denota uma crescente consciencialização da importância da inclusão da visão dos pacientes na segurança do paciente. No entanto, a maioria dos estudos identificados eram provenientes do Reino Unido ${ }^{2,27,29,32,38,41}$ e dos Estados Unidos da América $^{24,30,33,40}$, sendo que apenas dois dos estudos provinham de países não anglo-saxônicos ${ }^{34,35}$, pelo que seria interessante o desenvolvimento de estratégias de participação dos pacientes na segurança dos cuidados de saúde em outros países.

Nos estudos selecionados nesta revisão sistemática da literatura verificou-se que, tal como indica Saturno ${ }^{12}$, existe uma considerável variedade de estratégias e um grande avanço metodológico no âmbito da participação dos pacientes na melhoria contínua da segurança dos cuidados, tanto a nível de contribuir com informação para identificar problemas de segurança do paciente, como na colaboração ativa na prevenção de problemas. Contudo, como é possível verificar em alguns dos estudos selecionados, as diferentes estratégias podem ser complementares ${ }^{2,24,34,40}$.

A maioria das estratégias encontradas nesta revisão sistemática da literatura eram estratégias de solicitação ao paciente de informação sobre a segurança na prestação de cuidados (74\%), sendo as estratégias de mobilização dos utentes para a segurança dos cuidados de saúde e as estratégias para promover a participação ativa dos utentes na prevenção de incidentes de segurança, em número bastante inferior. Assim seria aconselhável centrar mais esforços em promover uma evolução validada e cientifica nestes tipos de estratégias.
Nesta revisão sistemática da literatura, foi possível verificar que, por exemplo, estratégias de solicitação ao paciente de informação sobre a segurança dos cuidados, como é o caso de questionários, estão a ser utilizadas para solicitar ao paciente diferentes tipos de informação sobre a segurança dos cuidados. Havendo questionários que têm como objetivo da sua aplicação o conhecimento da percepção de segurança, que os pacientes têm nos serviços/sistemas em que são aplicados ${ }^{24-26,37}$, e outros questionários que têm como finalidade da sua aplicação a identificação de problemas na segurança dos cuidados nos serviços sistemas em que são aplicados ${ }^{2,28,38,39}$. No entanto, estas duas tipologias de questionário são estratégias que os diferentes serviços/sistemas estão a utilizar e/ou desenvolver para integrar o "ponto de vista" do paciente na melhoria da segurança dos cuidados.

Através dos estudos selecionados pode também verificar-se que para situações descritas como semelhantes, se utilizaram diferentes tipos de estratégias, ou diferentes abordagens na utilização das mesmas, para incluir o paciente na melhoria da segurança dos cuidados, tornandose assim evidente a necessidade de estabelecer uma metodologia, estudada e aceite para a participação do paciente na segurança dos cuidados de saúde, tendo em conta o tipo de instituição, o âmbito de utilização, os problemas de segurança do paciente que apresentam e a disponibilidade financeira.

\section{Considerações finais}

Esta revisão sistemática permitiu verificar que existe descrita na literatura uma panóplia de estratégias que promovem a participação do paciente na segurança dos cuidados, que têm formas e métodos concretos de implementação, bem como objetivos distintos para a sua utilização. Assim, estas estratégias podem-se complementar umas às outras, dependendo das necessidades dos serviços/sistemas.

Entre as estratégias de promoção da participação dos pacientes na segurança dos cuidados de saúde, as que se encontram mais desenvolvidas e aplicadas em maior número, são as estratégias de solicitação ao paciente de informação sobre a segurança dos cuidados de saúde. Este tipo de estratégia foi aplicado em quinze dos dezenove estudos presentes nesta revisão. No entanto dentro destas, a estratégia mais utilizada foram os questionários, aplicados em oito estudos. 
As estratégias de promoção da participação dos pacientes na segurança dos cuidados de saúde podem ser utilizadas de forma individual ou complementar, sendo que é evidente a necessidade de estabelecer uma metodologia, para a elaboração e utilização adequada das estratégias de participação do paciente na segurança dos cuidados de saúde.

\section{Colaboradores}

FM Figueiredo trabalhou na conceção, delineamento, pesquisa e redação do artigo; AP Gálvez e AG Garcia trabalharam no delineamento da pesquisa, na metodologia e na redação do artigo; e M Eiras trabalhou na pesquisa e redação do artigo. 


\section{Referências}

1. Martin HM, Larsen J. Patient involvement in Patient Safety: A literature review about European primary care. Copenhagen: The Danish Institute for Health Services Research; 2012.

2. Ward JK, McEachan RR, Lawton R, Armitage G, Watt I, Wright J. Patient involvement in patient safety: Protocol for developing an intervention using patient reports of organisational safety and patient incident reporting. BMC Health Serv Res 2011; 11(1):130.

3. Sheard L, O’Hara J, Armitage G, Wright J, Cocks K, McEachan R, Watt I, Lawton R. Erratum to: Evaluating the PRASE patient safety intervention - a multi-centre, cluster trial with a qualitative process evaluation: study protocol for a randomised controlled trial. Trials 2016; 17(1):605.

4. de Vries EN, Ramrattan MA, Smorenburg SM, Gouma DJ, Boermeester MA. The incidence and nature of in-hospital adverse events: a systematic review. Qual Saf Heal Care 2008; 17(3):216-223.

5. Baker GR, Norton PG, Flintoft V, Blais R, Brown A, Cox J, Etchells E, Ghali WA, Hébert P, Majumdar SR, O’Beirne M, Palacios-Derflingher L, Reid RJ, Sheps S, Tamblyn R. The Canadian Adverse Events Study: the incidence of adverse events among hospital patients in Canada. CMAJ 2004; 170(11):1678-1686.

6. Kohn LT, Corrigan JM, Donaldson MS. To err is human: building a safer health system. Ann Fr Anesthesie Reanim 2000; 21(6):453-454.

7. World Health Organization (WHO). The Conceptual Framework for the International Classification for $\mathrm{Pa}$ tient Safety Version 1.1 Final Technical Report. 2009 [cited 2017 Oct 7]; 1(January):154. Available from: http://www.who.int/patientsafety/taxonomy/icps_ full_report.pdf

8. Hall J, Peat M, Birks Y, Golder S, Entwistle V, Gilbody S, Mansell P, McCaughan D, Sheldon T, Watt I, Williams B, Wright J. Effectiveness of interventions designed to promote patient involvement to enhance safety: a systematic review. BMJ Qual Saf 2010; 19(5):e10-e10.

9. The Health Foundation, Woolf SH, Kuzel AJ, Dovey SM, Phillips RL. Involving patients in improving safety (Evidence scan). Ann Fam Med [Internet]. 2013 [cited 2017 Dec 2]; 2(January):317-326. Available from: http://www.health.org.uk/sites/health/files/InvolvingPatientsInImprovingSafety.pdf

10. Longtin Y, Sax H, Leape LL, Sheridan SE, Donaldson L, Pittet D. Patient participation: current knowledge and applicability to patient safety. Mayo Clin Proc 2010; 85(1):53-62.

11. Souliotis K, Agapidaki E, Peppou LE, Tzavara C, Varvaras D. Original Article Assessing Patient Organization Participation in Health Policy: A Comparative Study in France and Italy. Kerman Univ Med Sci 2018; $7(1): 48-58$.

12. Saturno PJ. Estrategias para la participación del paciente en la mejora continua de la seguridad clínica. Rev Calid Asist [Internet]. 2009 Jun [cited 2017 Oct 7]; 24(3):124-130. Available from: http://linkinghub. elsevier.com/retrieve/pii/S1134282X09711418
13. Guadagnoli E, Ward P. Patient participation in decision-making. Soc Sci Med [Internet]. 1998 [cited 2017 Oct 7]; 47(3):329-339. Available from: http://www.sciencedirect.com/science/article/pii/S0 277953698000598

14. Levinson W, Kao A, Kuby A, Thisted RA. Not all patients want to participate in decision making. A national study of public preferences. J Gen Intern Med 2005; 20(6):531-535.

15. Doherty C, Stavropoulou C. Social Science \& Medicine Patients ' willingness and ability to participate actively in the reduction of clinical errors : A systematic literature review. Soc Sci Med 2012; 75(2):257-263.

16. Waterman $\mathrm{AD}$, Gallagher $\mathrm{TH}$, Garbutt J, Waterman BM, Fraser V, Burroughs TE. Brief report: Hospitalized patients' attitudes about and participation in error prevention. J Gen Intern Med [Internet]. 2006 [cited 2017 Oct 7]; 21(4):367-370. Available from: http://www.ncbi.nlm.nih.gov/pubmed/20042562\%0Ahttp://www.pubmedcentral.nih. gov/articlerender.fcgi?artid=PMC2800278 PMID: 16686815

17. Davis RE, Jacklin R, Sevdalis N, Vincent CA. Patient involvement in patient safety: What factors influence patient participation and engagement? [Internet]. Health Expectations 2007 [cited 2017 Oct 7]; 10(3):259-267. Available from: https://www.ncbi.nlm. nih.gov/pmc/articles/PMC5060404/pdf/HEX-10259.pdf PMID: 17678514

18. Entwistle VA, Mello MM, Brennan TA. Advising Patients About Patient Safety: current initiatives risk shifting responsibility. Jt Comm J Qual Patient Saf 2005; 31(9):483-494.

19. Watt PII, Birks Y, Entwistle V, Gilbody S, Hall J, Mansell P, Peat M, Sheldon T, Williams B. A review of strategies to promote patient involvement, a study to explore patient's views and attitudes and a pilot study to evaluate the acceptability of selected patient involvement strategies. Patient Saf Res Program PS/034. York: University of York; 2009.

20. Moher D, Liberati A, Tetzlaff J, Altman DG; PRISMA Group. Preferred Reporting Items for Systematic Reviews and Meta-Analyses: The PRISMA Statement. Ann Intern Med 2009; 151(4):264-269.

21. Higgins JPT, Green S. Manual Cochrane de revisiones sistemáticas de intervenciones. Cochrane [Internet]. 2011 [cited 2017 Oct 7]; (March):1-639. Available from: https://es.cochrane.org/sites/es.cochrane.org/ files/public/uploads/Manual_Cochrane_510_reduit. pdf PMID: 27699761

22. Higgins JPT, Altman DG, Gøtzsche PC, Jüni P, Moher D, Oxman AD , Savovic J, Schulz KF, Weeks L, Sterne JA; Cochrane Bias Methods Group; Cochrane Statistical Methods Group. The Cochrane Collaboration's tool for assessing risk of bias in randomised trials. BMJ 2011; 343:d5928.

23. Pope C, Mays JPN. Synthesizing qualitative and quantitative health evidence. Maiden Head: Open University/McGraw-Hill; 2007. 
24. Dixon JL, Tillman MM, Wehbe-Janek H, Song J, Papaconstantinou HT. Patients' Perspectives of Surgical Safety: Do They Feel Safe? Ochsner J [Internet]. 2015 [cited 2017 Oct 7]; 15(2):143-148. Available from: http://www.ncbi.nlm.nih.gov/pubmed/26130976

25. Mcinnes E, Chaboyer W, Murray E, Allen T, Jones P. The role of patients in pressure injury prevention: a survey of acute care patients. BMC Nurs 2014; 13(1):41.

26. Rathert C, Huddleston N, Pak Y. Acute care patients discuss the patient role in patient safety. Health Care Manage Rev 2011; 36(2):134-144.

27. Giles SJ, Lawton RJ, Din I, McEachan RRC. Developing a patient measure of safety (PMOS). BMJ Qual Saf 2013; 22(7):554-562.

28. Solberg LI, Asche SE, Averbeck BM, Hayek AM, Schmitt KG, Lindquist TC, Carlson RR. Can patient safety be measured by surveys of patient experiences? Jt Comm J Qual Patient Saf 2008; 34(5):266-274.

29. Hrisos S, Thomson R. Seeing It from Both Sides: Do Approaches to Involving Patients in Improving Their Safety Risk Damaging the Trust between $\mathrm{Pa}$ tients and Healthcare Professionals? PLoS One 2013; 8(11):e80759.

30. Anthony R, Miranda F, Mawji Z, Cerimele R, Davis R, Lawrence S. John M. Eisenberg Patient Safety Awards. The LVHHN patient safety video: patients as partners in safe care delivery. Jt Comm J Qual Saf [Internet]; 2003 Dec 1 [cited 2017 Oct 7]; 29(12):640-645. Available from: http://linkinghub.elsevier.com/retrieve/pii /S1549374103290754 PMID: 14679866

31. Mctier L, Botti M, Duke M. Patient participation in medication safety during an acute care admission. Heal Expect 2015; 18(5):1744-1756.

32. Pinto A, Vincent C, Darzi A, Davis R. A qualitative exploration of patients' attitudes towards the "participate inform notice know" (pink) patient safety video. Int J Qual Heal Care 2013; 25(1):29-34.

33. Millman EA, Pronovost PJ, Makary MA, Wu AW. Patient-assisted incident reporting: including the patient in patient safety. J Patient Saf 2011; 7(2):106-108.

34. Rodríguez MAP, Cerdá JCM, Suess A, Azarola AR, Terol E. Claraciones Y Convenios De Colaboración Con Las Comunidades Autónomas. Medicina Clinica 2008; 131(Supl. 3):33-38.

35. Jangland E, Carlsson M, Lundgren E, Gunningberg L. The impact of an intervention to improve patient participation in a surgical care unit: A quasi-experimental study. Int J Nurs Stud [Internet]. 2011 [cited 2017 Oct 7]; 49:528-538. Available from: https://www.sciencedirect.com/science/article/pii/S0020748911004214

36. Carter SR, Moles R, White L, Chen TF. Exploring patients' motivation to participate in Australia's Home Medicines Review program. Int J Clin Pharm 2012; 34(4):658-666.

37. Kistler CE, Walter LC, Mitchell CM, Sloane PD. Patient perceptions of mistakes in ambulatory care. Arch Intern Med 2010; 170(16):1480-1487.
38. Ricci-Cabello I, Pons-Vigués $\mathrm{M}$, Berenguera A, Pujol-Ribera E, Slight SP, Valderas JM. Patients' perceptions and experiences of patient safety in primary care in England. Fam Pract 2016; 33(5):535-542.

39. Hernan AL, Giles SJ, O'Hara JK, Fuller J, Johnson JK, Dunbar JA. Developing a primary care patient measure of safety (PC PMOS): a modified Delphi process and face validity testing. BMJ Qual Saf 2016; 25(4):273-280.

40. Brown M, Frost R, Ko Y, Woosley R. Diagramming patients' views of root causes of adverse drug events in ambulatory care: An online tool for planning education and research. Patient Educ Couns 2006; 62(3):302-315.

41. Ricci-Cabello I, Avery AJ, Reeves D, Kadam UT, Valderas JM. Measuring Patient Safety in Primary Care: The Development and Validation of the "Patient Reported Experiences and Outcomes of Safety in Primary Care" (PREOS-PC). Ann Fam Med 2016; 14(3):253-261.
Artigo apresentado em 24/05/2017 Aprovado em 04/05/2018 Versão final apresentada em 06/05/2018 
